\title{
RESPOSTAS FISIOLÓGICAS E ANATÔMICAS DE PLANTAS JOVENS DE EUCALIPTO EXPOSTAS AO CÁDMIO ${ }^{1}$
}

\author{
Teresa Cristina Lara Lanza de Sá e Melo Marques², Angela Maria Soares ${ }^{3}$, Marcelo Pedrosa Gomes ${ }^{4}$ e Giordane Martins ${ }^{5}$
}

\begin{abstract}
RESUMO - A poluição por metais pesados, particularmente pelo cádmio (Cd), é gerada principalmente pelas atividades de mineração e industrial, uso de adubos fosfatados e lodo de esgotos. Com o objetivo de avaliar o efeito do Cd em características fisiológicas e anatômicas, mudas de eucalipto foram plantadas em concentrações crescentes de Cd em solução nutritiva. As mudas de Eucalyptus camaldulensis permaneceram durante 20 dias em vasos de $1 \mathrm{~L}$ contendo solução nutritiva de Clark, sendo os tratamentos de 0, 15, 25, 45 e $90 \mu \mathrm{mol} \mathrm{Cd} \mathrm{L}^{-1}$ fornecido como $\mathrm{CdSO}_{4}$. Durante o período experimental, foram realizadas avaliações de estado hídrico e de teores de pigmentos nas datas 1, 3, 6, 12 e 20 dias após indução dos tratamentos (DAT). As avaliações de produção de fitomassa, teores de Cd e características anatômicas foram feitas com material vegetal coletado no final do período experimental. Houve redução na produção de matéria seca, mesmo nas menores concentrações do elemento. Os teores de clorofila diminuíram com o aumento da concentração de Cd aos 20 DAT, enquanto os

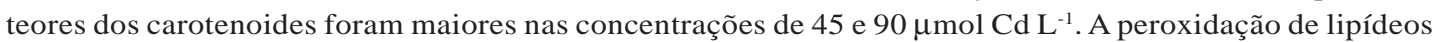
nas folhas foi crescente com o aumento da concentração do metal aos 20 DAT, enquanto nas raízes o efeito do Cd foi pouco acentuado. O potencial hídrico aumentou em todas as concentrações de Cd aos 12 e 20 DAT. As espessuras dos tecidos radiculares também aumentaram com o aumento das doses de Cd, porém as do mesofilo e do limbo foliar diminuíram. Portanto, os resultados obtidos evidenciam que o tempo de exposição e maiores doses de Cd acentuam os danos ocorridos em E. camaldulensis para a maioria das características avaliadas. Algumas alterações observadas revelam o potencial de tolerância de plantas de E. camaldulensis à exposição ao Cd.

Palavras-chaves: Solução nutritiva, Tolerância e Fitorremediação.
\end{abstract}

\section{PHYSIOLOGICAL AND ANATOMICAL RESPONSES OF EUCALYPTUS SEEDLINGS EXPOSED TO CADMIUM}

\begin{abstract}
Pollution caused by heavy metals, mainly by cadmium (Cd), is generated by mining and industrial activities, and by the use of phosphated fertilizers and sewage sludge in agriculture. Aiming to investigate the Cd effects on physiological and anatomical features, eucalyptus seedlings in Cd-increasing concentrations in nutrient solution. Seedlings of Eucalyptus camaldulensis remained for 20 days in $1 \mathrm{~L}$ vases with Clark solutions, and the

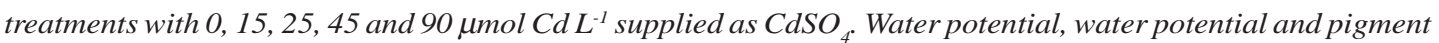
content evaluations were carried out on dates 1, 3, 6, 12 and 20 days after treatments induction (DAE). Evaluations of mass production, Cd contents and anatomical characteristics were made with vegetal matter collected in the end of the experimental period. There was a decrease on dry matter production, even in the lowest Cd concentration. Total chlorophyll content decreased with the increase of Cd content in nutrient solution at 20 days after exposure

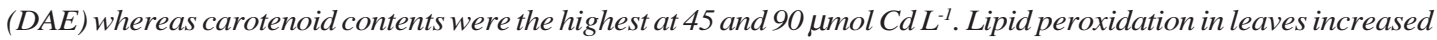
with increasing Cd levels at 20 DAE, whereas in root, the effect of Cd was less pronunciated. Water potential increased at all Cd concentration at 12 and 20 DAE. Root tissues thickness also increased increased as Cd levels increased, but mesophyll and leave blade thickness decreased. Thus, the results indicate that the exposure time and higher doses of Cd accentuate damages in $\boldsymbol{E}$. camaldulensis for most of the evaluated traits. Some changes observed reveal the tolerance potential of $\boldsymbol{E}$. camaldulenses to Cd-exposition.
\end{abstract}

Keywords: Nutrient solution, Tolerance and Phytoremediation.

\footnotetext{
${ }^{1}$ Recebido em 12.03.2009 e aceito para publicação em 02.05.2011.

${ }^{2}$ Departamento de Ciência do Solo da Universidade Federal de Lavras, UFLA, Brasil. E-mail: <tmarques004@hotmail.com>. ${ }^{3}$ Departamento de Biologia, Setor de Fisiologia da Universidade Federal de Lavras, UFLA, Brasil. E-mail: <amsoares@dbi.ufla.br> .

${ }^{4}$ Departamento de Biologia, Setor de Fisiologia Vegetal da Universidade Federal de Lavras, UFLA, Brasil. E-mail: <marcelopgom@yahoo.com.br>.

${ }^{5}$ Departamento de Biologia, Setor de Fisiologia Vegetal da Universidade Federal de Lavras, UFLA, Brasil. E-mail: <giordanemartins@gmail.com>.
} 


\section{INTRODUÇÃO}

Por ser um elemento de alta mobilidade e toxicidade, o Cd apresenta elevado potencial de risco ambiental (ARDUINI et al., 1996), justificando assim estudos que possam contribuir para o desenvolvimento de técnicas que visam a recuperação de áreas contaminadas. A fitorremediação é uma técnica alternativa para essa recuperação, porém é um processo limitado pela eficiência de aquisição e translocação dos metais para os diferentes órgãos das plantas e pelas diferentes capacidades de tolerância e acúmulo desses metais nas espécies vegetais.

Vários estudos têm mostrado o Cd como inibidor de processos fisiológicos na planta, entre esses a fotossíntese, que é afetada através da interferência do metal na síntese de clorofila (PRASAD, 1995; HORVÁTH et al., 1996), na organização dos cloroplastos (SANDALIO et al., 2001) e em outras fases do processo (CLIJSTERS; VANASSCHE, 1985; CHUGH; SAWHNEY, 1999). A redução no crescimento como resposta ao aumento dos níveis fitotóxicos indicam claramente um impacto ecofisiológico dos metais pesados às plantas (MONNI et al., 2001).

Modificações em características fisiológicas e anatômicas têm sido observadas em muitos estudos de fitorremediação, como consequência da capacidade da espécie vegetal em se adaptar ao ambiente estressante (BARCELÓ; POSCHENREIDER, 1990; CHUGH; SAWHNEY, 1999; MONNI et al., 2001). As espécies reativas de oxigênio (EROs) produzidas pelas plantas quando expostas ao Cd causam danos de membrana. Entretanto, espécies vegetais são capazes de aumentar a atividade de enzimas antioxidantes como forma de reduzir os danos causados pelas EROs, aumentando assim a tolerância ao Cd em seus tecidos (SINGH et al., 2006). Alterações na arquitetura e estrutura do sistema radicular são observadas em plantas cultivadas na presença de metais pesados, bem como submetidas a diferentes condições de salinidade (LUX et al., 2004; REINHARDT et al., 1995;WÓJCIK et al., 2005). Também se observam alterações anatômicas da parte aérea em plantas expostas ao Cd através do acréscimo de concentração de materiais fenólicos na parede celular e no lúmen do xilema (VOLLENWEIDER et al., 2006) e redução no tamanho das células paliçádicas e da epiderme (SRIDHAR et al., 2005).

Devido ao seu rápido crescimento e à capacidade de acumular metais pesados em seus tecidos, algumas espécies de Eucalyptus têm sido consideradas potenciais para a fitorremediação de áreas contaminadas por metais, como o Cd (ACCIOLY et al., 2004). O entendimento dos processos fisiológicos e das características morfoanatômicas associadas à extração de poluentes do ambiente é de grande importância para a compreensão e melhorias no processo de fitorremediação. Sendo assim, este trabalho teve como objetivo avaliar o acúmulo de fitomassa, teores de pigmentos, potencial hídrico, a peroxidação lipídica e as características anatômicas de raízes e folhas de plantas de Eucalyptus camaldulensis submetidas a diferentes doses de Cd em solução nutritiva.

\section{MATERIAL E MÉTODOS}

O experimento foi realizado em casa de vegetação do Departamento de Ciência do Solo da Universidade Federal de Lavras (UFLA), no Município de Lavras, MG. Sementes de Eucalyptus camaldulensis, oriundas de área de produção comercial de semente em Açailândia - MA, foram colocadas para germinar em bandeja de isopor e, após 45 dias, as mudas foram transferidas para bandejas contendo solução nutritiva de Clark (CLARK, 1975), permanecendo por 15 dias. Após este período de adaptação, as mudas foram selecionadas quanto à altura e transferidas para vasos de $1 \mathrm{~L}$ de solução nutritiva, sendo aplicados os tratamentos, que constituíram de cinco doses de Cd (0, 15, 25, 45 e $90 \mu \mathrm{mol} \mathrm{L}^{-1}$ ) na forma de $\mathrm{CdSO}_{4}$, com quatro repetições por tratamento. Durante o período experimental de 20 dias, foram realizadas avaliações de estado hídrico e de teor de pigmentos nas datas 1, 3, 6, 12 e 20 dias após indução dos tratamentos (DAT).

O estado hídrico das plantas foi avaliado por meio de medidas do potencial hídrico de base ( $\Psi_{\mathrm{W}}$ máximo), realizadas sempre antes do amanhecer, com auxílio de uma câmara de pressão (Soil Moisture Equipment Corp - Modelo 3005, Santa Barbara, CA-USA), em folhas completamente expandidas do $3^{\circ}$ ou $4^{\circ}$ nó a partir do ápice para a base da planta. Para a avaliação dos pigmentos e peroxidação de lipídeos, folhas completamente expandidas foram coletadas entre o ápice e o meio da planta, sendo imediatamente acondicionadas em papel de alumínio e colocadas em caixa de isopor com gelo. O método de quantificação das clorofilas a, b e total foi realizado segundo Arnon (1949). Para a determinação dos carotenoides, empregou-se o método de Duke e Kenyon (1986), quantificando os teores do $\beta$-caroteno (SANDMANN; BÖRGER, 1983). 
Para a peroxidação de lipídeos das folhas e raízes, empregou-se o método de quantificação de malonildialdeído (MDA) através da produção de metabólitos reativos a ácido 2-tiobarbitúrico (TBA), baseado nos trabalhos de Heath e Packer (1968) e Buege e Aust (1978). Ao final de 20 dias, as plantas foram colhidas, separadas em raízes e parte aérea, colocadas em sacos de papel e levadas a estufa com circulação constante de ar a $70^{\circ} \mathrm{C}$ até massa constante para determinação de matéria seca.

As avaliações anatômicas foram feitas com material vegetal coletado no final do período experimental. Raízes foram coletadas e fixadas em solução Karnovsky (glutaraldeído (2,5\% m/v) e paraformaldeído $(2,5 \% \mathrm{~m} / \mathrm{v}))$ por 48 horas. Posteriormente, iniciou-se o processo de desidratação em gradiente alcoólico (30\%, 40\%, 50\%, 60\%, 70\%, $80 \%, 90 \%$ e $100 \%$ duas vezes por 1 hora), incluídas em série crescente butanol/historresina 25\% (Historesin, Leica) (2 horas), 40\% (2 horas) e 100\% (por 5 dias), sendo os espécimes montados em moldes e polimerizados em temperatura ambiente, e secções transversais de $15 \mu \mathrm{m}$ foram feitas com auxílio de micrótomo rotativo. As secções foram coradas com azul de toluidina $0,3 \%(\mathrm{~m} / \mathrm{v})$ em tampão de tetraborato de sódio 1\% (m/v, pH 9) e montadas em lâminas de vidro.

Para as avaliações atômicas foliares, folhas completamente expandidas do $3^{\circ}$ ou $4^{\circ}$ nó do ápice para a base foram coletadas e fixadas em FAA 70 (formaldeído + ácido acético + álcool etílico 70\%) (JOHANSEN, 1940). Foram realizadas secções paradérmicas na região mediana da folha e coradas com safranina $1 \%(\mathrm{~m} / \mathrm{v})$. Seç̧ões transversais foram feitas à mão livre no terço médio foliar, clarificados em água sanitária 5\%(KRAUS; ARDUIM, 1997) e coradas com azul de astra e safranina. As lâminas foram montadas em glicerina 50\%.

A análise e a documentação fotográfica do laminário histológico foram realizadas utilizando-se um microscópio fotônico (Ken-a-Vision TT18, New York, EUA) com câmera digital (Canon Power Shot A620, Tokyo, Japão).

Para a obtenção dos dados quantitativos referentes à espessura da epiderme e endoderme radicular, bem como da densidade estomática (número de estômatos $\mathrm{mm}^{-2}$ ) e densidade de células epidérmicas propriamente ditas das folhas (número de células $\mathrm{mm}^{-2}$ ), foram realizadas cinco observações por planta, em campos com área correspondente a $0,067 \mathrm{~mm}^{2}$, com auxílio do software Sigma Scan. O cálculo do índice estomático foi feito de acordo com a expressão de Cutter (1986): índice estomático $(\mathrm{IE})=[\mathrm{NE} /(\mathrm{CE}+\mathrm{NE})] \times 100$, em que NE é o número de estômatos e CE o número de células epidérmicas propriamente ditas.

Os resultados obtidos foram submetidos à análise de variância (Sigma Plot). Erro padrão da média (EPM) foi também determinado para cada média estudada. As médias das características anatômicas foram comparadas pelo teste de Scott-Knott, a 5\%, empregando-se o programa Sisvar.

\section{RESULTADOS E DISCUSSÃO}

O acúmulo de matéria seca das raízes e parte aérea foi proporcionalmente reduzido com o aumento dos teores de Cd na planta (Figura 1), quando estas foram submetidas ao metal em solução nutritiva. Foi verificada redução de mais de 50\% na produção de matéria seca de raízes e da parte aérea no tratamento de $90 \mu \mathrm{mol}$ $\mathrm{Cd} \mathrm{L}^{-1}$, em relação ao controle. A razão entre a matéria seca das raízes e da parte aérea (R/PA) para os

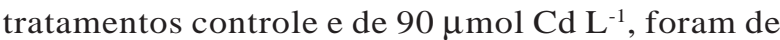
0,36 e 0,37 , respectivamente. Reduções na biomassa de raízes e parte aérea ocorreram de maneira igual pelo excesso de Cd. Dados semelhantes foram encontrados por Soares et al. (2005) para as espécies E. maculata e E. urophilla, também utilizando solução nutritiva. Diversos estudos relatam que o Cd afeta processos metabólicos como fotossíntese, respiração e atividade de enzimas associadas ao sistema antioxidante das plantas (HORVÁTH et al., 1996; SANDALIO et al., 2001; SINGH et al., 2006). Esses efeitos causam a redução do nível energético da planta, levando à diminuição da capacidade de absorção de micronutrientes e água, afetando consequentemente o crescimento.

A razão clorofila a/b não diferiu entre os tratamentos controle e na presença de Cd apenas no $1^{\circ} \mathrm{DAT}$ (Tabela 1 ). No $3^{\circ}$ e $12^{\circ}$ DAT, a razão clorofila a/b foi maior nos tratamentos com Cd, que no controle. No $6^{\circ}$ e $20^{\circ} \mathrm{DAT}$, o controle apresentou maior razão que os tratamentos que receberam Cd, sendo o tratamento de $90 \mu \mathrm{mol} \mathrm{Cd} \mathrm{L}{ }^{-1}$ o que apresentou menor valor. Essa diminuição pode ser explicada devido à diminuição nos teores de clorofila a (dados não apresentados) como resposta ao excesso de Cd. Alterações nos teores de clorofila devido à toxidez 


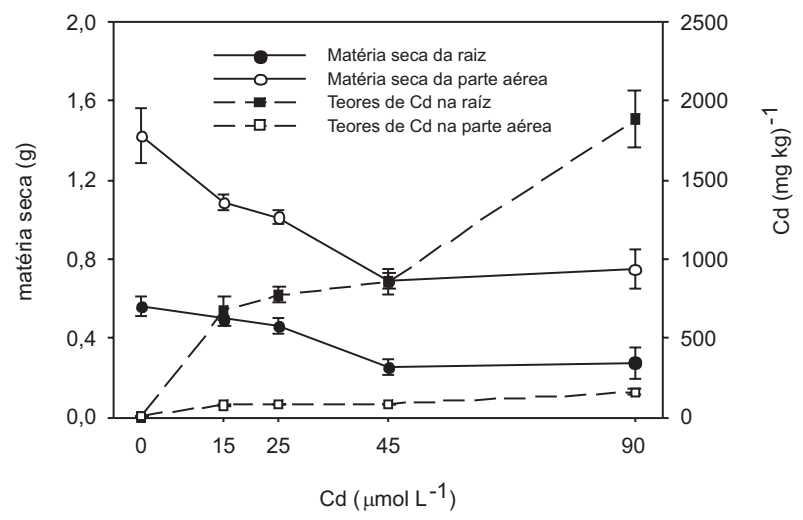

Figura 1 - Produção de matéria seca de raízes e parte aérea e os teores de Cd na matéria seca de raízes e parte aérea de plantas de E. camaldulensis, submetidos a várias doses de Cd, em solução nutritiva, durante 20 dias. Cada ponto representa a média \pm erro padrão de três repetições.

Figure 1 - Root and shoot dry matter productions and Cd concentrations on root and shoot dry matter of E. camaldulensis, subjected to several Cd levels, in nutrient solution, for 20 days. Each dot represent means \pm standard error for three replicates. de Cd são relatadas em vários estudos (HORVÁTH et al., 1996; DI CAGNO et al., 1999; CHUGH; SAWHNEY, 1999). Segundo Stobart et al. (1985), os metais pesados interferem na biossíntese da clorofila através da inibição da formação do ácido $\alpha$-aminolevulínico (ALA), e também da inibição da redutase do protoclorofilídio, a enzimachave da fototransformação do protoclorofilídioclorofílidio. Na Tabela 1, verifica-se também o efeito significativo do tempo de exposição das plantas ao excesso de Cd no teor de clorofila total. Aos 12 e 20 DAT, observou-se maior redução no teor de clorofila total com a exposição das plantas às doses crescentes de Cd, sendo que aos 20 DAT, este efeito foi mais acentuado. Em plântulas de Helianthus annus, as folhas mais velhas tiveram o teor de clorofila $a$ e clorofila total reduzidos, enquanto a clorofila b e a razão clorofila a/b não foram observado nenhuma alteração (DI CAGNO et al., 1999).

O Cd afetou pouco a concentração dos carotenoides em relação ao controle (Tabela 1), sugerindo alguma capacidade da planta em tolerar $\mathrm{Cd}$ em seus tecidos. Ao final do experimento, verifica-se que houve uma

Tabela 1 - Razão clorofila a/b, teores de clorofila total e carotenóides em plantas jovens de E. camaldulensis, submetidas a doses crescentes de $\mathrm{Cd}\left(\mu \mathrm{mol} \mathrm{L}{ }^{-1}\right)$ em solução nutritiva, em diferentes épocas de coletas (DAT), durante 20 dias.

Table 1 - Chlorophyll a/b ratio, total chlorophyll and carotenoid contents in E. camaldulensis seedlings subjected to increasing doses of $\mathrm{Cd}\left(\mu \mathrm{mol} \mathrm{L} \mathrm{L}^{-1}\right)$ in nutrient solution and in different collect dates (DAE), for 20 days.

\begin{tabular}{|c|c|c|c|c|c|c|c|c|c|c|}
\hline$\overline{\mathrm{DAT}}$ & 1 & & 3 & & 6 & & 12 & & 20 & \\
\hline$\mu \mathrm{mol} \mathrm{L}-1$ & & \multicolumn{9}{|c|}{ Razão clorofila a/b } \\
\hline 0 & 3,06 & $\pm 0,009$ & 2,49 & $\pm 0,005$ & 2,97 & $\pm 0,002$ & 2,26 & $\pm 0,004$ & 3,58 & $\pm 0,002$ \\
\hline 15 & 3,03 & $\pm 0,007$ & 3,17 & $\pm 0,005$ & 2,83 & $\pm 0,001$ & 2,83 & $\pm 0,021$ & 3,44 & $\pm 0,004$ \\
\hline 25 & 2,93 & $\pm 0,008$ & 2,56 & $\pm 0,002$ & 2,85 & $\pm 0,004$ & 2,61 & $\pm 0,007$ & 3,24 & $\pm 0,004$ \\
\hline 45 & 2,94 & $\pm 0,007$ & 3,81 & $\pm 0,008$ & 2,86 & $\pm 0,003$ & 2,60 & $\pm 0,010$ & 3,04 & $\pm 0,009$ \\
\hline 90 & 3,13 & $\pm 0,004$ & 3,06 & $\pm 0,002$ & 2,66 & $\pm 0,013$ & 2,76 & $\pm 0,010$ & 3,04 & $\pm 0,005$ \\
\hline$\mu \mathrm{mol} \mathrm{L}{ }^{-1}$ & & \multicolumn{9}{|c|}{ Clorofila total (mg g $\left.{ }^{-1} \mathrm{MF}\right)$} \\
\hline 0 & 1,17 & $\pm 0,001$ & 1,40 & $\pm 0,001$ & 1,57 & $\pm 0,001$ & 1,69 & $\pm 0,001$ & 1,61 & $\pm 0,001$ \\
\hline 15 & 1,19 & $\pm 0,001$ & 1,43 & $\pm 0,001$ & 1,64 & $\pm 0,001$ & 1,60 & $\pm 0,002$ & 1,48 & $\pm 0,001$ \\
\hline 25 & 1,61 & $\pm 0,001$ & 1,27 & $\pm 0,001$ & 1,58 & $\pm 0,001$ & 1,63 & $\pm 0,001$ & 1,33 & $\pm 0,001$ \\
\hline 45 & 1,21 & $\pm 0,001$ & 1,20 & $\pm 0,001$ & 1,56 & $\pm 0,001$ & 1,40 & $\pm 0,001$ & 1,32 & $\pm 0,002$ \\
\hline 90 & 1,41 & $\pm 0,001$ & 1,32 & $\pm 0,002$ & 1,58 & $\pm 0,003$ & 1,62 & $\pm 0,001$ & 1,25 & $\pm 0,001$ \\
\hline$\mu \mathrm{mol} \mathrm{L}{ }^{-1}$ & & \multicolumn{9}{|c|}{ Carotenóides $\left(\mu \mathrm{g} \mathrm{g}^{-1} \mathrm{MF}\right)$} \\
\hline 0 & 53,22 & $\pm 0,037$ & 51,48 & $\pm 0,473$ & 73,30 & $\pm 0,254$ & 40,56 & $\pm 7,052$ & 21,44 & $\pm 0,821$ \\
\hline 15 & 42,10 & $\pm 0,011$ & 48,52 & $\pm 0,516$ & 63,13 & $\pm 0,992$ & 39,53 & $\pm 5,806$ & 45,30 & $\pm 1,095$ \\
\hline 25 & 57,79 & $\pm 0,032$ & 46,63 & $\pm 0,620$ & 59,15 & $\pm 5,482$ & 25,86 & $\pm 1,259$ & 36,36 & $\pm 0,490$ \\
\hline 45 & 51,48 & $\pm 1,243$ & 63,47 & $\pm 5,995$ & 56,29 & $\pm 0,604$ & 72,05 & $\pm 0,636$ & 39,27 & $\pm 1,815$ \\
\hline 90 & 64,11 & $\pm 5,368$ & 68,37 & $\pm 2,179$ & 56,31 & $\pm 1,568$ & 35,12 & $\pm 1,764$ & 49,03 & $\pm 0,028$ \\
\hline
\end{tabular}

Todos os valores são médias \pm erro-padrão de três repetições.

Data are means \pm SD for three replicates.

Revista Árvore, Viçosa-MG, v.35, n.5, p.997-1006, 2011 
tendência dos teores de carotenoides aumentarem em relação à data anterior. Entretanto para o tratamento

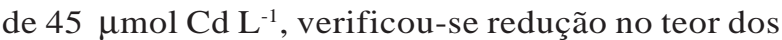
carotenóides aos 20 DAT. Na maior dose de Cd e aos 20 DAT, verifica-se que houve um acréscimo na concentração do pigmento de $129 \%$ em relação ao controle. Aumentos nos teores de carotenoide nas plantas, geralmente estão relacionados com o aumento da tolerância ao estresse oxidativo (SOMASHEKARAIAH et al., 1992; SINGH et al., 2006).

De modo geral, os teores de MDA nas folhas foram crescentes com o aumento da concentração de Cd e com o tempo de exposição ao metal (Figura 2). Maiores efeitos do Cd na peroxidação de lipídeo das folhas foram verificados nos tratamentos que receberam 45 e $90 \mu \mathrm{mol} \mathrm{Cd} \mathrm{L-1}$, em média, superiores em $22 \%$ e $43 \%$ em relação ao controle, respectivamente. Este efeito foi agravando-se com o aumento do tempo de exposição ao metal.

O efeito do $\mathrm{Cd}$ nos tecidos radiculares afetou os teores de MDA de modo geral a reduzir seus valores com o aumento da concentração do metal (Figura 2). Os tratamentos 15 e $25 \mu \mathrm{mol} \mathrm{Cd} \mathrm{L}{ }^{-1}$ resultaram em menores reduções na peroxidação de lipídeos das raízes, reduzindo em média 7\% e 16\%, respectivamente, em relação ao controle. Provavelmente, com menores concentrações do Cd em solução, as EROs foram eliminadas por enzimas antioxidantes, reduzindo os danos na membrana celular de raízes de $E$. camaldulensis. Em doses superiores, a peroxidação de lipídeos nas membranas de raízes foi, em média, bem próxima daquela ocorrida no tratamento-controle. Isto sugere que, mesmo sendo as raízes o órgão acumulador de $\mathrm{Cd}$, este foi capaz de desenvolver mecanismo de suportar altas concentrações do metal em seus tecidos, sem grandes danos.

O tempo de exposição ao Cd também alterou o potencial hídrico das plantas (Figura 3). Até o $6^{\circ} \mathrm{DAT}$, os tratamentos que receberam $\mathrm{Cd}$ apresentaram potencial hídrico menor que o controle. Após essa data, houve diminuição no potencial hídrico das plantas dos

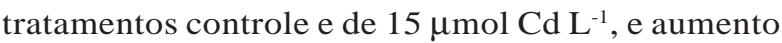
nos demais tratamentos. Os maiores valores do potencial hídrico observados com o aumento da concentração de Cd podem ser associados à menor taxa de transpiração devido à menor produção de matéria seca.
A redução nas taxas de transpiração pode ser também decorrente dos efeitos do Cd na resistência estomática. Redução na taxa de transpiração ou aumento na resistência estomática têm sido observados em plantas expostas ao Cd (BARCELÓ; POSCHENREIDER, 1990). Em Arabidopsis thaliana, o efeito do Cd na relação água-planta foi verificado através da redução na condutância foliar induzida pela ação direta do Cd no fechamento dos estômatos (PERFUS-BARBEOCH et al., 2002).

O Cd afetou significantemente a espessura dos tecidos radiculares. Verificou-se aumento na espessura da epiderme e endoderme radicular proporcional ao aumento nas doses de Cd (Tabela 2). Por apresentar acúmulo de cargas negativas, o aumento na espessura da epiderme pode ampliar o papel de filtro biológico para os íons metálicos exercido por esse tecido. $\mathrm{O}$ aumento em espessura da endoderme, uma barreira apoplástica, pode representar menor translocação do Cd para a parte aérea, diminuindo os efeitos tóxicos deste metal no sistema fotossintético. Modificações de espessura e constituição química das barreiras apoplásticas são adaptações importantes para a tolerância das plantas a fatores de estresse.

As espessuras do mesofilo e do limbo foliar diminuíram de acordo com o aumento nas doses de $\mathrm{Cd}$, não tendo diferenciado entre os tratamentos 15 e $25 \mu \mathrm{mol} \mathrm{L}{ }^{-1} \mathrm{Cd}$ e entre os tratamentos 45 e $90 \mu \mathrm{mol} \mathrm{L}{ }^{-1} \mathrm{Cd}$ (Tabela 2). A diminuição da espessura do mesofilo sugere uma redução na capacidade fotossintética das plantas expostas ao Cd, o que tem sido verificado em vários estudos (BASZYNSKI et al., 1980; CHUGH; SAWHNEY, 1999; DI CAGNO et al., 1999). Sirdhar et al. (2005) verificaram o colapso de células dos parênquimas paliçádico e lacunoso de Brassica juncea exposta a Zn e Cd. Tais dados poderiam explicar a redução nas espessuras do mesofilo e do limbo foliar verificadas neste estudo. A espessura das epidermes abaxial e adaxial da folhas foi menor nos tratamentos 45 e $90 \mu \mathrm{mol} \mathrm{L}^{-1} \mathrm{Cd}$, que não diferiram entre si (Tabela 2). Em experimentos com feijão, a expansão celular foi inibida e o teor relativo de água foi reduzido, sugerindo que o $\mathrm{Cd}$ induziu a redução na extensibilidade celular (POSCHENREIDER et al., 1989), o que poderia, em parte, explicar a diminuição da epiderme foliar nos tratamentos de maior dose de $\mathrm{Cd}$. O número de células

Revista Árvore, Viçosa-MG, v.35, n.5, p.997-1006, 2011 


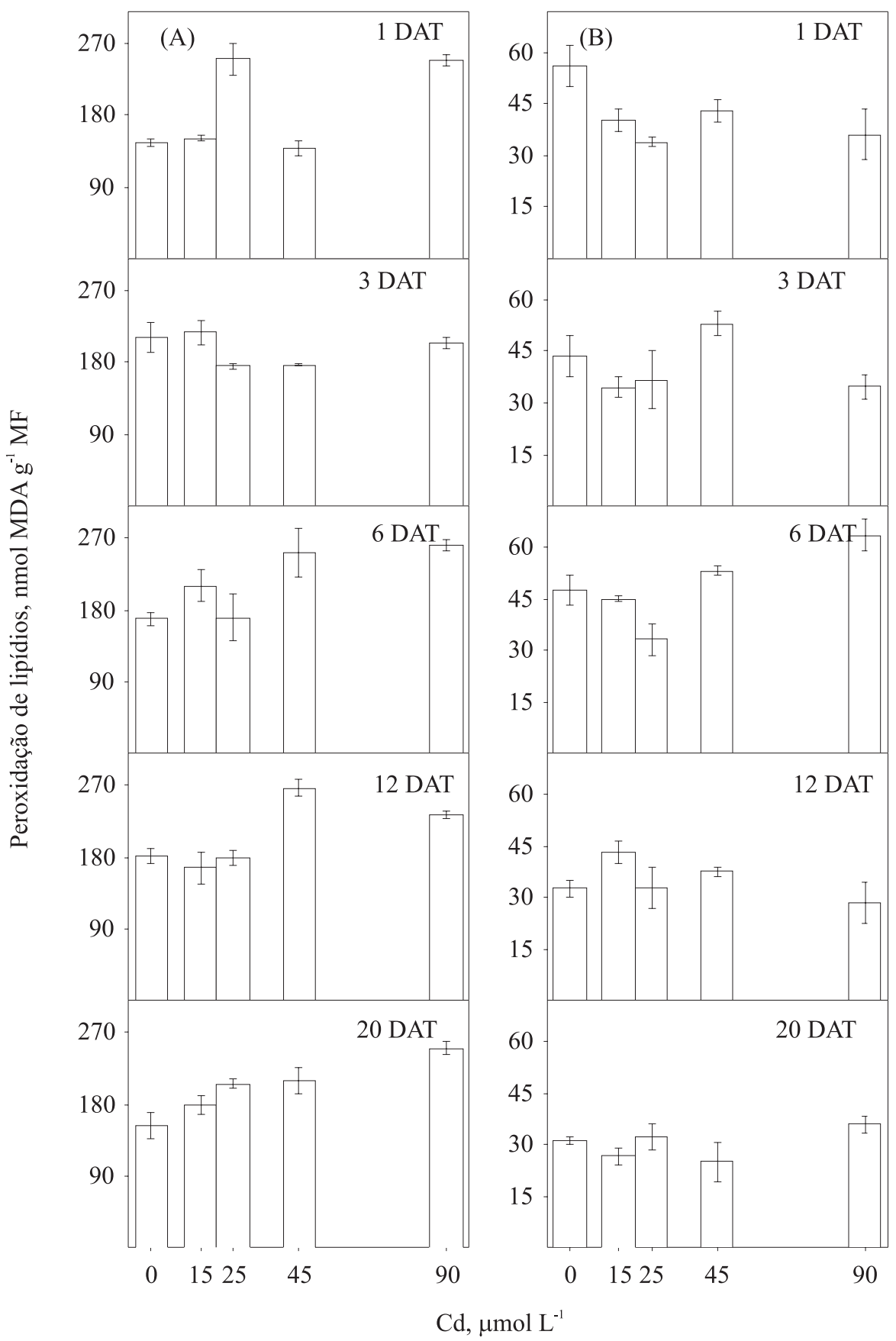

Figura 2 - Peroxidação de lipídeos nas folhas (A) e raízes (B) de plantas jovens de E. camaldulensis em diferentes épocas de coleta (DAT) e em várias doses de Cd, em solução nutritiva, durante 20 dias. Cada ponto representa a média \pm erro-padrão de 3 repetições.

Figure 2 - Lipid peroxitation in leaves (A) and roots (B) of $\boldsymbol{E}$. camaldulensis seedlings on different collect dates (DAE) and exposed to several Cd levels, in nutrient solution for 20 days. Each dot represent means \pm standard error for three replicates.

Revista Árvore, Viçosa-MG, v.35, n.5, p.997-1006, 2011 


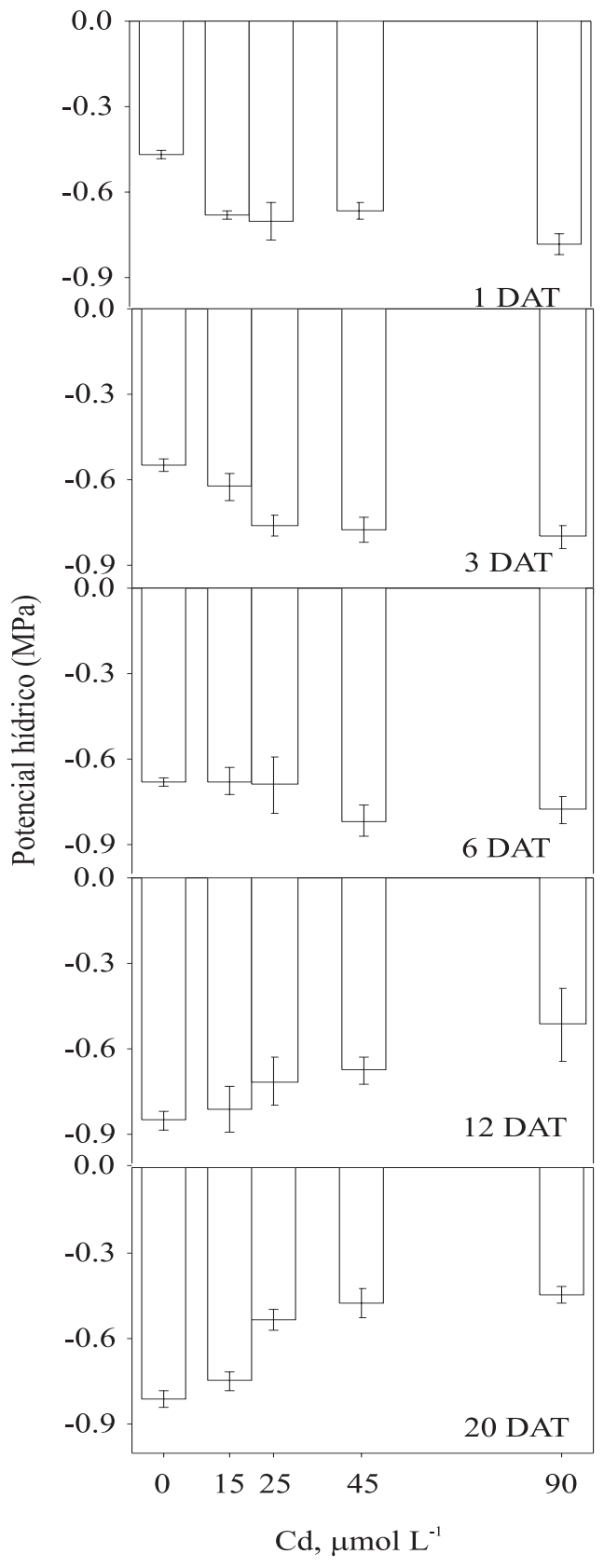

Figura 3 - Potencial hídrico (MPa) de plantas jovens de E. camaldulensis, em diferentes épocas de coleta (DAT) e em várias doses de Cd, em solução nutritiva, durante um período de 20 dias. Cada ponto representa a média \pm erro padrão de 3 repetições.

Figure 3-Water potential (MPa) of E. camaldulensis seedlings on different collect dates (DAE) and exposed to several Cd levels in nutrient solution for 20 days. Each dot represents means \pm standard error for three replicates. das epidermes da face abaxial e adaxial das folhas aumentou proporcionalmente com o aumento das doses de Cd na solução, não diferindo entre os tratamentos controle e $15 \mu \mathrm{mol} \mathrm{L}{ }^{-1} \mathrm{Cd}$ na epiderme da face abaxial (Tabela 2). O índice estomático de ambas as faces da epiderme foi menor no tratamento $90 \mu \mathrm{mol} \mathrm{L}{ }^{-1} \mathrm{Cd}$ (Tabela 2). Considerando que o índice estomático é um atributo que permite obter informações sobre a diferenciação celular de maneira independente do processo de expansão celular (TICHÁ, 1982), pode-se dizer que o $\mathrm{Cd}$ influencia o processo de diferenciação de células do meristemoide para formação de estômatos, e consequentemente reduz a taxa de diferenciação.

A densidade estomática das epidermes das faces abaxial e adaxial teve aumento proporcional ao aumento das doses de Cd na solução (Tabela 2). Os diâmetros polar e equatorial dos estômatos de ambas as faces não diferiram entre os tratamentos (Tabela 2). Aumento na densidade estomática de folhas em condições de estresse por Cd tem sido verificado em Silene vulgaris (CHARDONNENS et al., 1998), Brassica napus (BARYLA et al., 2001) e Brassica juncea (SALT et al., 1995). A análise desses resultados, juntamente com os obtidos para o índice estomático, permite afirmar que o Cd, além de afetar a taxa de diferenciação, influencia o processo de expansão celular, diminuindo o tamanho das células.

\section{CONCLUSÃO}

Os resultados obtidos indicam que plantas de E. camaldulensis.apresentam respostas em nível fisiológico e anatômico quando expostas ao Cd. O tempo de exposição e maiores doses de Cd acentuam os danos ocorridos em E. camaldulensis para a maioria das características avaliadas. Há modificações na produção de matéria seca, na peroxidação de lipídeos e nas relações hídricas das plantas em função do aumento da dose de $\mathrm{Cd}$, sendo os efeitos mais acentuados em maiores doses. O aumento no teor de carotenoides indica mecanismos de proteção ao Cd em relação aos danos por estresse oxidativo. As alterações na anatomia das raízes, com aumento na espessura da endoderme e epiderme, revelam também o potencial de adaptação de plantas de $E$. camaldulensis à exposição ao $\mathrm{Cd}$.

Revista Árvore, Viçosa-MG, v.35, n.5, p.997-1006, 2011 
Tabela 2 - Densidade, índice estomático e espessura de células e tecidos de plantas jovens de E. camaldulensis submetidas a doses crescentes de Cd na solução nutritiva, durante 20 dias.

Table 2 - Stomatal density and index and thickness of cells and tissues of $\mathbf{E}$. camaldulensis seedlings exposed to increasing Cd levels in nutrient solution for 20 days.

\begin{tabular}{|c|c|c|c|c|c|}
\hline \multirow[t]{2}{*}{ Características Avaliadas } & \multicolumn{5}{|c|}{ Tratamentos $\left(\mu \mathrm{mol} \mathrm{Cd} \mathrm{\textrm {L } ^ { - 1 } )}\right.$} \\
\hline & 0 & 15 & 25 & 45 & 90 \\
\hline Epid. radicular $(\mu \mathrm{m})$ & 10,08 e & $16,58 \mathrm{~d}$ & 20,49 c & $25,34 \mathrm{~b}$ & 30,16 a \\
\hline Endod. radicular $(\mu \mathrm{m})$ & $11,37 \mathrm{e}$ & $13,60 \mathrm{~d}$ & $15,48 \mathrm{c}$ & $17,40 \mathrm{~b}$ & 25,10 a \\
\hline Mesofilo foliar $(\mu \mathrm{m})$ & 292,40 a & $273,50 \mathrm{~b}$ & $272,60 \mathrm{~b}$ & $208,30 \mathrm{c}$ & 209,70 c \\
\hline Limbo foliar $(\mu \mathrm{m})$ & 326,75 a & $312,20 \mathrm{~b}$ & 309,50 b & $236,40 \mathrm{c}$ & $241,20 \mathrm{c}$ \\
\hline Epiderme abaxial $(\mu \mathrm{m})$ & 16,80 a & $17,20 \mathrm{a}$ & 17,20 a & $11,35 \mathrm{~b}$ & $13,80 \mathrm{~b}$ \\
\hline Epiderme adaxial $(\mu \mathrm{m})$ & $18,00 \mathrm{a}$ & $21,70 \mathrm{a}$ & $19,80 \mathrm{a}$ & $15,90 \mathrm{~b}$ & $17,70 \mathrm{~b}$ \\
\hline N Cel. EAb. & $1509,20 \mathrm{~d}$ & $1510,30 \mathrm{~d}$ & 1596,80 с & $1787,90 \mathrm{~b}$ & $2249,10 \mathrm{a}$ \\
\hline $\mathrm{N}^{\circ}$ Cel. EAd. & 1204,00 e & $1373,90 \mathrm{~d}$ & $1627,00 \mathrm{c}$ & $1699,60 \mathrm{~b}$ & 2050,90 a \\
\hline Dens. Est. Ad. & 326,80 e & $240,50 \mathrm{~d}$ & $416,50 \mathrm{~b}$ & $388,70 \mathrm{c}$ & 457,30 a \\
\hline DPEEAb $(\mu \mathrm{m})$ & 21,40 a & $22,10 \mathrm{a}$ & 20,30 a & 18,90 a & 18,30 a \\
\hline DEEEAb $(\mu \mathrm{m})$ & $15,20 \mathrm{a}$ & 15,60 a & 13,30 a & 13,50 a & 13,40 a \\
\hline Dens. Est. Ab. & 187,40 e & $231,60 \mathrm{~d}$ & 270,90 с & 283,60 a & $274,80 \mathrm{~b}$ \\
\hline DPEEAd $(\mu \mathrm{m})$ & 22,10 a & $22,50 \mathrm{a}$ & 22,60 a & 22,20 a & 20,00 a \\
\hline DEEEAd $(\mu \mathrm{m})$ & $14,30 \mathrm{a}$ & $14,60 \mathrm{a}$ & $14,60 \mathrm{a}$ & $12,70 \mathrm{a}$ & $12,70 \mathrm{a}$ \\
\hline IEEAb & 17,79 с & $18,39 \mathrm{~b}$ & 20,68 a & $17,85 \mathrm{c}$ & $16,89 \mathrm{~d}$ \\
\hline IEEAd & $13,46 \mathrm{~b}$ & 14,42 a & $14,27 \mathrm{a}$ & 14,29 a & $11,81 \mathrm{c}$ \\
\hline
\end{tabular}

Epid. radicular = epiderme radicular; Endod. radicular $=$ endoderme radicular; $\mathrm{N}^{\circ} \mathrm{Cel}$. EAb $=\mathrm{n}^{\circ}$ de células da epiderme abaxial; $\mathrm{N}^{\circ} \mathrm{Cel}$ EAd. $=n^{\circ}$ de células da epiderme adaxial; Dens. Est. Ad. = densidade estomática da epiderme adaxial $\left(\mathrm{n}^{\circ} / \mathrm{mm}^{2}\right) ; \mathrm{DPEEAd}=\mathrm{diâmetro}$ polar dos estômatos da epiderme adaxial; DEEEAd = diâmetro equatorial do estômatos da epiderme adaxial; Dens. Est. Ab. = densidade estomática da epiderme abaxial $\left(\mathrm{n}^{\circ} / \mathrm{mm}^{2}\right)$; DPEEAb = diâmetro polar dos estômatos da epiderme abaxial; DEEEAb = diâmetro equatorial do estômatos da epiderme abaxial; IEEAb = índice estomático da epiderme abaxial; IEEAd = índice estomático da epiderme adaxial. Médias seguidas pela mesma letra, na linha, não diferem estatisticamente entre si, pelo teste Scott-Knott a $\mathrm{P} \leq 0,05$ ( $\mathrm{n}=5$ ).

Epid. radicular $=$ root epidermis; Endod. radicular $=$ root endodermis; $N^{\circ}$ Cel. EAb $=$ number of abaxial epidermis cells; $N^{\circ}$ Cel. EAd. = number of abaxial epidermis cells; Dens. Est. Ad. = stomatal density of adaxial epidermis $\left(n^{\circ} / \mathrm{mm}^{2}\right) ; \mathrm{DPEEAd}$ = stomatal polar diameter on adaxial epidermis; DEEEAd = equatorial diameter of stomata on adaxial epidermis; Dens. Est. Ab. = stomatal density of abaxial epidermis $\left(n^{\circ} / \mathrm{mm}^{2}\right) ; D P E E A b=$ polar diameter of stomata on abaxial epidermis; $D E E E A b=$ equatorial diameter of stomata on abaxial epidermis; IEEA $b=$ stomatal index of abaxial epidermis; IEEAd = stomatal index of adaxial epidermis.

\section{REFERÊNCIAS}

ACCIOLY, A. M. A. et al. Amenização do calcário na toxidez de zinco e cádmio para mudas de Eucalyptus camaldulensis cultivadas em solo contaminado.

Revista Brasileira de Ciência do Solo, v.28, n.4, p.775-783, 2004.

ARDUINI, J.; GODBOLD, D. L.; ONNIS, A. Cadmium and copper uptake and distribution in Mediterranean tree seedlings. Physiologia Plantarum, v.97, n.1, p.111-117, 1996.

ARNON, D. I. Cooper enzymes in isolated chloroplasts. Polyphenoloxidase in Beta vulgaris. Plant Physiology, v.24, n.1, p.1-15, 1949.

BARYLA, A. et al. Leaf cloroses in oilseed rape plants (Brassica napus) grown o cadmiumpolluted soil: causes and consequences for photosynthesis and growth. Planta, v.212, n.5/6, p.696-709, 2001
BARCELÓ, J.; POSCHENRIEDER, C. Plant water relations as affected by heavy metal stress: A review. Journal of Plant Nutrition, v.13, n.1, p.1-37, 1990.

BASZYNSKI., T. et al. Photosynthetic activities of cadmium - treated tomato plants. Physiologia Plantarum, v.48, n.3, p.365-370, 1980.

BUEGE, J. A.; AUST, S. D. Microsomal lipid peroxidation. Methods Enzymology, v.52, p.302, 1978.

CHARDONNENS, A. N. et al. Distribution of cadmium in leaves of cadmium tolerant and sensitive ecotypes of Silene vulgaris. Physiologia Plantarum, v.104, n.1, p.75-80, 1998.

CHUGH, L. K.; SAWHNEY, S. K. Photosynthetic activities of Pisum sativum seedlings grown in presence of cadmium. Plant Physiology and Biochemistry, v.37, n.4, p.297-303, 1999. 
CLIJSTERS, H.; van ASSCHE, F. Inhibition of photosynthesis by heavy metals. Photosynthetic Research, v.7, n.1, p.31-40, 1985.

CLARK, R. B. Characterization of phosphates in intact maize roots. Journal of Agriculture and Food Chemistry, v.23, n.3, p.458-460,1975.

CUtTER, E. G. Anatomia vegetal. Parte I Células e tecidos. 2.ed. São Paulo: Roca, 1986. 304p.

DI CAGNO, R. et al. Effects of cadmium on growth of Helianthus annus seedlings: physiological aspects. New Phytologist, v.14, n.1, p.65-71, 1999.

DUKE, S. O.; KENYON, W. H. Effects of dimethazone (FMC) on chloroplast development. II. Pigment synthesis and photosynthesis function in cowpea (Vigna unguiculata L.). Pesticide Biochemistry and Physiology, v.25, n.1, p.11-18, 1986.

HEATH, R. L.; PACKER, L. Photoperoxidation in isolated chloroplasts. I. Kinetics and stoichiometry of fatty acid peroxidation.

Archives of Biochemistry and Biophysics, v.125, n.1, p.189-198, 1968.

HORVÁTH, G. et al. Formation of the photosynthetic apparatus during greening of cadmium-poisoned barley leaves. Planta, v.99, p.238-243, 1996.

JOHANSEN, D. A. Plant microtechinique. 2.ed. New York: MC GRAW-HILL, 1940.

KRAUS, J. E.; ARDUIN, M. Manual básico de métodos em morfologia vegetal. Seropédica: EDUR, 1997.

LUX, A. A. et al. Differences in struture of adventitions roots in Salix clones with contrasting characteristics of cadmium accumulation and sensitivity. Physiologia Plantarum, v.120, n.4, p.537-545, 2004.

MONNI, S. et al. Ecophysiological responses of Empetrum nigrum to heavy metal pollution. Environmental Pollution, v.112, n.1, p.121-129, 2001.
PERFUS-BARBEOCH, L. et al. Heavy metal toxicity: cadmium permeates through calcium channels and disturbs the plant water status. The Plant Journal, v.32, n.4, p.539-548, 2002.

POSCHENREIDER, C.; GUNSÉ, B.; BARCELÓ, J. Influence of cadmium on water relations, stomatal resistence, and abscisic acid content in expanding bean leaves. Plant Physiology, v.90, n.4 p.1365-1371, 1989.

PRASAD, M. N. V. Cadmium toxicity and tolerance in vascular plants. Environmental and Experimental Botany, v.35, n.1, p.525-545, 1995.

REINHARDT, D. H.; ROST, T. L. Salinity accelerates endodermal development and induces an exodermis in cotton seedling roots.

Environmental and Experimental Botany, v.35, n.4, p.563-674, 1995.

SALT, D. E. et al. Mechanisms of cadmium mobility and accumulation in Indian Mustard. Plant Physiology, v.109, n.4, p.1427-1433, 1995.

SANDALIO, M. et al. Cadmium-induced changes in the growth and oxidative metabolism of pea plants. Journal of Experimental Botany, v.52, n.364, p.2115-2126, 2001.

SANDMANN, G.; BÖGER, P. Comparison of the bleaching activity of norflurazon and oxyfluorfen. Weed Science, v.31, n.3, p.338-341, 1983.

SINGH, S.; EAPEN, S.; D’SOUZA, S. F. Cadmium accumulation and its influence on lipid peroxidation and antioxidative system in an aquatic plant, Bacopa monnieri L.

Chemosphere, v.62, n.2, p.233-241, 2006.

SRIDHAR, B. B. M. et al. Anatomical changes due to uptake and accumulation of $\mathrm{Zn}$ and $\mathrm{Cd}$ in Indian mustard (Brassica juncea).

Environmental and Experimental Botany, v.54, n.2, p.131-141, 2005

SOARES, C. R. F. S. et al. Fitotoxidez de cádmio para Eucalyptus maculata e E. uruphylla em solução nutritiva. Revista Árvore, v.29, n.1, p.175-183, 2005.

Revista Árvore, Viçosa-MG, v.35, n.5, p.997-1006, 2011 
SOMASHEKARAIAH, B. V.; PADMAJANA, K.;

PRASAD, A. R. K. Phytotoxicity of cadmium ion on germinating seedlings of mung bean (Phaseolus vulgaris): involvement of lipid peroxides in chlorophyll degradation. Physiologia

Plantarum, v.85, n.1, p.85-89, 1992.

STOBART, A. K. et al. The effect of $\mathrm{Cd}^{2+}$ on the biosynthesis of chlorophyll in leaves of barley. Physiologia Plantarum, v.63, n.3, p.293-298, 1985.

TICHÁ, I. Photosynthetic characteristics during ontogenesis of leaves: stomatal density and size. Photosynthetica, v.16, n.3, p.375-471, 1982.
VOLLENWEIDER, P. et al. Localization and effects of cadmium in leaves of a cadmium-tolerant willow (Salix viminalis L.). Part II - Microlocalization and cellular effects of cadmium.

Environmental and Experimental

Botany, v.58, n.1, p.25-40, 2006.

WÓJCIK, M. et al. Cadmium tolerance in Thlaspi caerulescens. Environmental and Experimental Botany, v.53, n.1, p.163-171, 2005. 